\title{
Estrategias de aprendizaje y eLearning. Un apunte para la fundamentación del diseño educativo en los entornos virtuales de aprendizaje.
}

\author{
Learning Strategies and E-Learning. Some Notes on the \\ Foundations of Learning Design in Virtual Learning \\ Environments.
}

\author{
Manuel Esteban-Albert \\ Universidad de Murcia, Murcia, España \\ mesteban@um.es \\ Miguel Zapata-Ros \\ Universidad de Murcia, Murcia, España \\ mzapata@um.es
}

\section{Resumen}

En este artículo se abordan conceptos y realidades de vigencia como son las estrategias y estilos de aprendizaje, así como el concepto de metacognición. Además se plantea desde el punto de vista de si pueden servir para fundamentar el diseño educativo en actividades de eLearning y en entornos virtuales de aprendizaje (EVA).

Se pone de relieve el papel de la metacognición en el eLearning sobre todo pensando en la selección, secuenciación y organización de los contenidos atendiendo a las características y situaciones de aprendizaje específicas de los alumnos. El carácter autodidáctico y favorecedor del aprendizaje autónomo que poseen estos entornos parece requerir, más que ningún otro, de un buen conocimiento de los propios recursos para aprender. Pero, ¿es posible, o fácil, inducir elementos que incrementen esta ineludible e intransferible función del aprendiz situado en estos entornos? ¿Cómo o con qué herramientas cognitivas o de regulación del propio conocimiento? ¿Bajo qué criterios o condiciones se podrían incorporar en las actividades de Elearning elementos de control de la actividad cognitiva de los sujetos? ¿Las guías didácticas y particularmente las especificaciones del learning design pueden cumplir esas funciones? ¿Bajo qué criterios o condiciones?

Estas son las preguntas que nos planteamos.

En cuanto a los estilos de aprendizaje, se plantea: $\mathrm{Si}$, con la información que disponemos acerca de ellos, seríamos capaces de definir los rasgos de un estilo de aprendizaje apropiado para los EVA, o más bien si es necesario tenerlos en cuenta en el diseño instruccional. ¿Existe pues la suficiente cultura en la dirección de observar y conocer los estilos de aprender de los alumnos para orientar la enseñanza en la dirección de mejorar las condiciones de aprendizaje de los EVA o hacerlos al menos posible? ¿Cuáles pueden ser ingredientes esenciales de los entornos de aprendizaje, y del diseño instruccional, para llevar a cabo esa función?

Palabras clave: Metacognición, estilos de aprendizaje, estrategias de aprendizaje, eLearning, entornos virtuales de aprendizaje, diseño educativo, objetos de aprendizaje, SCORM.

\footnotetext{
Abstract

This article deals with concepts and topics of present relevance: learning styles and strategies, metacognition, and their role in the foundation of the learning design of elearning activities and virtual learning environments. (VLE).
} 


\begin{abstract}
The importance of metacognition in e-learning stands out, basically taking into account the selection, sequencing and organization of contents which pay particular attention to students' specific characteristics and learning situations. The qualities of promoting selfstudy and autonomy that these environments have seem to need, more than others, a sound knowledge of the student's own learning resources. However, is it easy or feasible to include elements to increase these resources, which pertain unavoidably and in a nontranferable way to the student's self? How, or which are the cognitive or regulating tools of one's own knowledge that are to be used? Under which criteria or conditions could elements to control the cognitive activity of the individuals be incorporated in e-learning activities? Could this role be fulfilled by didactic guides or by specifications in learning design? Under which criteria and conditions? - these are the questions we pose.

As regards learning syles, we wonder if the information available is enough to define the features of an apropriate learning style for VLE, or rather, if it's necesary to take them into account for the instructional design. So, do we observe and learn enough about the students' learning styles so as to direct teaching towards better learning conditions in the VLE, or make them posible, at least? Which may be the essential elements in the VLE and in the instructional design to carry out this function?
\end{abstract}

Key words: Metacognition, learning styles, learning strategies, eLearning, learning design, virtual learning environments, instructional design, learning objects, SCORM.

\title{
Introducción
}

En el número 7 de RED apareció, en febrero de 2003, el artículo titulado Las estrategias de aprendizaje en el entorno de la Educación a Distancia. Consideraciones para la reflexión y el debate. Introducción al estudio de las estrategias y estilos de aprendizaje, de Manuel Esteban (Esteban, 2003) como publicación de un material de los cursos a distancia de capacitación docente virtual que impartíamos desde la Universidad de Murcia. Este trabajo viene siendo accedido con carácter relativamente intenso y regular desde entonces, según documenta el servicio de estadística de accesos de la revista.

Por el interés manifestado por los lectores hemos creído conveniente actualizarlo e insertarlo en las coordenadas que definen las tendencias del elearning, con ideas ya expuestas en trabajos anteriores aparecidos en RED (Zapata, 2005) y en las demandas que recientemente se ha planteado la unión europea sobre "aprender a aprender" como competencia básica (Zapata y Cerezo, Mayo 2007).

En el presente artículo pues, se abordan conceptos y realidades de vigencia en la psicología del aprendizaje, como son las estrategias y estilos de aprendizaje, así como el concepto de metacognición, y se plantean algunas cuestiones que puedan servir para fundamentar aspectos del diseño educativo en actividades de eLearning y en los entornos virtuales de aprendizaje (EVAs).

Sin entrar en un tratamiento en profundidad ni exhaustivo, se pone de relieve el papel de la metacognición en el contexto del eLearning y en general de los entornos virtuales de aprendizaje (EVAs), pensando sobre todo en ideas que fundamenten y guíen la práctica del diseño educativo en estos entornos, sobre todo en la selección, secuenciación y organización de los contenidos atendiendo a las características y situaciones de aprendizaje específicas de los alumnos. El carácter autodidáctico que, en gran medida, poseen estos entornos parece requerir, más que ningún otro, de un buen conocimiento de los propios recursos para aprender. Pero, ¿es posible, o fácil, inducir elementos que incrementen esta ineludible e intransferible función del aprendiz situado en EVAs? ¿Cómo o con qué herramientas cognitivas o de regulación del propio conocimiento? 
¿Bajo qué criterios o condiciones se podrían incorporar en las actividades de Elearning elementos de control de la actividad cognitiva de los sujetos? ¿Las guías didácticas y particularmente las especificaciones del learning design pueden cumplir esas funciones? ¿Bajo qué criterios o condiciones?

Estas son las preguntas que nos planteamos y sobre las que llamamos la atención.

En cuanto a los estilos de aprendizaje se plantean otras cuestiones: Si, con la información que disponemos acerca de ellos, seríamos capaces de definir los rasgos de un estilo de aprendizaje apropiado, ideal, para los EVAs, lo cual nos induciría en unos planteamientos escasamente inclusivistas, o más bien si es necesario tenerlos en cuenta en el diseño instruccional. ¿Existe pues la suficiente cultura en la dirección de observar y conocer los estilos de aprender de los alumnos para orientar la enseñanza en la dirección de mejorar las condiciones de aprendizaje de los EVAs o hacerlos al menos posibles? ¿Cuáles pueden ser ingredientes esenciales de los entornos de aprendizaje, y del diseño instruccional, para llevar a cabo esa función?

La Comisión Europea presenta los principios, objetivos y líneas de acción del eLearning como «la utilización de las nuevas tecnologías multimediales y de Internet, para mejorar la calidad del aprendizaje facilitando el acceso a recursos y servicios, así como los intercambios y la colaboración a distancia» ${ }^{1}$. Esta definición es a nuestro modo de ver la que, sin comprometerse excesivamente con ninguna de las opciones en presencia, da cabida a la mayoría de las conceptualizaciones existentes y desde luego alberga, o al menos no excluye, la acción como eje central de la intervención directa de los agentes implicados, en calidad de tutores, coordinadores, moderadores, organizadores didácticos, de recursos, etc. Y desde luego la que despierta mayor consenso.

Sin embargo la práctica de los medios especializados ---foros, listas de discusión, literatura especializada, etc.---, primero en U.S.A. y luego en el resto del mundo, ha acuñado de forma laxa este término para referirse al mundo de la formación y de la educación que en algún momento, o en la mayoría de los momentos, utiliza las redes y los medios digitales como soporte de su actividad.

También se ha consolidado el uso del término E-learning empresarial, en sentido más limitado, para referirse a una industria y a un negocio, y a su correlato en el mundo de la investigación tecnológica, que ha tenido como objetivo distribuir conocimientos soportados en multimedia (multimedia educativo o en la web) con objeto de ser aprendidos por un sector más o menos preciso de destinatarios. Se trata de lo que comúnmente se conoce como el e-learning empresarial (Ruíperez, 2003) o del sector empresarial, que pone en circulación materiales de aprendizaje más o menos asistidos por un auténtico sistema de aprendizaje en redes. En realidad es esta la auténtica esencia del E-learning para muchos, donde se distingue la distribución de contenidos más o menos asistidos (se entiende asistidos de forma automática) y el sentido instrumental de éstos en un contexto educativo. Se trata en caso extremo del negocio del e-learning, concepto incluido dentro de lo que se llaman las industrias de la información.

En este contexto surgen los objetos de aprendizaje o learning objets (Wiley, 2002)

\footnotetext{
${ }^{1}$ Iniciativa «eLearning - Concebir la educación del futuro» el 24 de mayo de 2000 http://europa.eu.int/comm/education/programmes/elearning/index_es.html
} 
¿Qué son pues los objetos de aprendizaje? Desde el punto de vista de la intervención psicopedagógica: unidades curriculares soportadas digitalmente que pueden integrarse en distintos contextos curriculares apoyando programas formativos con distintos objetivos y destinatarios.

Pero sobre todo se trata de asegurar unos objetivos comunes de accesibilidad, interoperabilidad, durabilidad y reutilización de los materiales curriculares basados en las redes.

Naturalmente hay otras acepciones. Sin embargo, si aceptamos ésta, el problema de la industria del e-learning se centra en la ausencia de unas metodologías psicopedagógicas comunes y consensuadas que garanticen estos objetivos.

En este complejo mundo se han desarrollado dos áreas para abordar el problema: la de los estándares de intercambio de datos (SCORM) y la del diseño instruccional tecnológico, es decir el desarrollo de especificaciones que sobre la base de una supuesta excelencia pedagógica permita a los desarrolladores de software elaborar aplicaciones educativas de calidad.

La forma que proponen y habilitan los técnicos y teóricos del ELearning para resolver el problema del intercambio de datos son los estándares de ELearning: Protocolos que contienen las especificaciones para dotar de flexibilidad a las propuestas de teleformación para su transferencia, tanto en el formato de los materiales y su estructura, así como en la conformación de las infraestructuras (herramientas informáticas y telemáticas). Y que está dando resultados en los aspectos tecnológico y documental. Particularmente SCORM ${ }^{2}$.

Según esta idea los estándares han iniciado el camino hacia una forma cómoda y viable de empaquetar los recursos y contenidos, tanto para los estudiantes que cambian de sistema, para los docentes que utilizan en distintos contextos estos materiales, como para los desarrolladores que tienen que construir nuevas herramientas y mejorar las vigentes.

A esto se le une en el rubro del "haber" el que los productos que eventualmente se adhieran a estos estándares se les asegura que no quedarán obsoletos a corto o a medio plazo. De esta forma se protegen las inversiones realizadas. La economía también se produce en el terreno del conocimiento. Así se prevé en los medios del eLearning empresarial. Los estándares comunes para los metadata de los materiales, el empaquetamiento y secuencia de los recursos, la interoperabilidad de herramientas, ahorran inversión en aprendizajes adicionales y esfuerzos de diseño docente.

En este rubro, el de los logros, también se quiere introducir la elaboración, justificación y definición de especificaciones que permitan diseñar soportes digitales de aplicaciones educativas (Units of Learning, $\mathrm{UoL}$ ).

\footnotetext{
${ }^{2}$ SCORM (Sharable Content Object Reference Model, Modelo de Referencia para Objetos de Contenidos Intercambiables): Los materiales bajo estándar SCORM deben asegurar el cumplimiento de cuatro principios:

1. Reusabilidad: Que los objetos de aprendizaje puedan ser reutilizados en distintas plataformas y en distintos contextos tecnológicos y curriculares. Y en qué grado se puede hacer.

2. Accesibilidad: Que las herramientas y productos SCOPRM permitan un seguimiento del comportamiento y del historial de aprendizaje y académico de los alumnos

3. Interoperabilidad: Que se pueda intercambiar información a través de todas las plataformas (LMS.) que adopten los estándares

4. Durabilidad: Que los productos SCORM eviten la obsolescencia de los materiales.
} 
La elaboración, justificación y definición de especificaciones que permitan diseñar aplicaciones educativas está liderado por una corporación de la industria del eLearning, IMS Global Learning Consortium.

Pero ¿qué es?, ¿cómo funciona?

Griffiths. Blat, García y Sayazo (Griffiths et al., 2005) lo explican perfectamente en el artículo de monográfico 4 de $\mathrm{RED}^{\mathrm{i}}$ :

En 1997 la Open University of the Netherlands (OUNL) decidio convertir todos sus cursos en cursos on-line. Los cursos existentes empleaban una variedad de enfoques pedagógicos. La Universidad los clasificó y empezó a implementar unas plantillas representativas para intentar dar soporte a todas estas categorías pedagógicas. Rápidamente se constató que todos los profesores tenían su propia visión pedagógica, y que necesitaban casi tantas plantillas como profesores. Por otro lado, aunque había muchas descripciones pedagógicas de los cursos, en la práctica todas consistían en combinaciones de tres elementos básicos: recursos educativos, múltiples personas actuando en varios roles, y actividades pedagógicas. El EML (Educational Modelling Language), introducido por la OUNL, permite definir estos tres elementos y así especificar la estructura de una Unit of Learning, UoL, mediante un documento $X M L$.

(...)

IMS, consciente de las limitaciones pedagógicas de las especificaciones existentes, empezó el proceso de desarrollo de una especificación para la definición de aspectos pedagógicos, pero ya que EML existía y funcionaba decidieron adaptarlo en lugar de crear una especificación totalmente nueva. El resultado es una nueva especificación, IMS Learning Design. Aunque presenta cambios importantes de estructura y enfoque, sus conceptos básicos y capacidades son muy similares a los de EML.

(...) los aspectos importantes de IMS Learning Design son los siguientes:

ofrece soporte para múltiples alumnos, y contempla la comunicación entre ellos

* representa el papel de profesor

permite combinar recursos educativos con actividades pedagógicas, y con las interacciones entre personas en diferentes roles.

Estas capacidades facilitan que el diseñador de las UoL pueda definir, por ejemplo, actividades de aprendizaje del tipo problem-based Learning (enseñanza basada en problemas).

Las especificaciones que el presumible y deseable, según los autores, estándar IMSGlobal Consortium (IMS-GC) ofrece sobre otras alternativas están en que ellos ofrecen exclusivamente las herramientas de ejecución necesarias para implementar los más variados principios pedagógicos. La cuestión radica en que IMS-GC, en un proceso previo, ya había subsumido el proceso de decantación de estos principios en una fase anterior e interna, sin sometimiento a otras condiciones o contextos formativos: El lenguaje fue desarrollado originalmente en la universidad abierta de los Países Bajos (OUNL), después de un examen y de una comparación extensos de una amplia gama de itinerarios pedagógicos y sus actividades de aprendizaje asociadas, y de refinamientos 
sucesivos del lenguaje tendentes a obtener un buen equilibrio entre la generalidad y la expresividad pedagógica.

En general el procedimiento es claro: Obtener una información supuestamente basada en principios de calidad pedagógica, en algunos casos pero no siempre ni lo más frecuente, y en la mayor parte de las veces a partir de un conocimiento experto, estudio de casos, etc. pero casi nunca en desarrollos completos de teorías o de principios generales de intervención psicopedagógica (la psicología del aprendizaje en principio y en cualquier circunstancia debería establecer sistemas inductivos/deductivos y en general debería definir estructuras sistémicas que describieran cómo se producen los aprendizajes diferenciándolos, y relacionando contenidos, condiciones y métodos, y no operar exclusivamente en principios o en procedimientos acabados), y a continuación aplicarlos directamente en la fase de diseño y desarrollo de productos tecnológicos y en técnicas de programación. Obteniendo productos orientados en una dirección: que cada vez sean más autónomos de lo que es una intervención docente directa.

Esta forma de operar a veces tiene éxitos, como es el caso de las pautas para la accesibilidad, es decir en obtener las especificaciones para un diseño instruccional accesible ${ }^{\text {ii. }}$

Sin embargo, en el rubro del "debe", de las expectativas no satisfechas, se constatan varios hechos: este propósito se hace difícilmente compatible con los principios de la secuenciación de contenidos y con las leyes que rigen el entramado cognitivo que existe en los aprendices y permite la incorporación de conocimientos, como ya vimos con anterioridad (Zapata, 2006, pp. 4 a 6 y Zapata, 2005, pp. 30 a 34). Y en particular, como planteamos ahora, con los elementos de singularidad cognitiva de los estudiantes ¿cómo perciben y se representan el conocimiento?, las habilidades o competencias metacognitivas y los estilos y estrategias cognitivas.

Como es sabido, el concepto de estrategias se incorpora recientemente a la psicología del aprendizaje y la educación como una forma más de resaltar el carácter procedimental que tiene todo aprendizaje. Es tanto como afirmar que los procedimientos usados para aprender son una parte muy decisiva del resultado final de ese proceso. No es que se ignorase, sobre todo por los buenos "maestros", la importancia decisiva de las técnicas y otros recursos aportados por el aprendiz pero no existía una formulación y conceptualización tan explícita y con términos específicos sobre esas tales operaciones cognitivos del aprendiz. Es, pues, un concepto moderno que conecta adecuadamente con los principios de la psicología cognitiva, con la perspectiva constructivista del conocimiento y aprendizaje, con la importancia atribuida a los elementos procedimentales en el proceso de construcción de conocimientos $\mathrm{y}$, asimismo, con los aspectos diferenciales de los individuos tan enfatizados por toda la psicología cognitiva (adultos, jóvenes, expertos, novatos, etc.).

Resaltaremos, a modo de resumen, algunos de los aspectos que pueden resultar más relevantes. El concepto de estrategia implica una connotación finalista e intencional. Toda estrategia ha de ser un plan de acción ante una tarea que requiere una actividad cognitiva que implica aprendizaje. No se trata, por tanto, de la aplicación de una técnica concreta, por ejemplo aplicar un método de lectura (SQ3R u otro). Se trata de un dispositivo de actuación que implica habilidades y destrezas -que el aprendiz ha de poseer previamente- y una serie de técnicas que se aplican en función de las tareas a 
desarrollar. Quizás lo más importante de esta consideración es que para que haya intencionalidad ha de existir conciencia de: a) la situación sobre la que se ha de operar (problema a resolver, datos a analizar, conceptos a relacionar, información a retener, etc.).

De donde resulta, desde el punto de vista del aprendizaje, muy importante la representación de la tarea que se hace el aprendiz en la toma de decisión sobre las estrategias a aplicar; y b) de los propios recursos con que el aprendiz cuenta, es decir, de sus habilidades, capacidades, destrezas, recursos y de la capacidad de generar otros nuevos o mediante la asociación o reestructuración de otros preexistentes.

En definitiva, esta conciencia de los propios recursos cognitivos con que cuenta el aprendiz, que los psicólogos llamamos pedantemente metacognición, no es sólo una estrategia o conjunto de estrategias de diverso orden. En nuestra opinión, es condición necesaria para que pueda darse cualquier plan estratégico ya que de lo contrario podría darse la aplicación de estrategias, cierto, pero no habría intencionalidad al no existir la adopción de un plan con previa deliberación de la situación y los recursos.

Las estrategias se suelen clasificar, generalmente, en función de las actividades cognitivas a realizar. Atendiendo a ese criterio se suelen clasificar, desde las operaciones más elementales a las más elaboradas en asociativas, de elaboración, de organización. Las primeras, las más simples, implican operaciones básicas y elementales que no promueven en sí mismas relaciones entre conocimientos pero pueden ser la base para su posterior elaboración ya que incrementan la probabilidad de recordar literalmente la información, sin introducir cambios estructurales en ella. Las estrategias de elaboración constituyen un paso intermedio entre las estrictamente asociativas que no trabajan la información en sí misma y las de organización que promueven nuevas estructuras de conocimiento. En la elaboración se pueden producir operaciones más simples donde se establecen algunas relaciones, por lo general extrínsecas, entre elementos de la información que pueden servir de "andamiaje" al aprendizaje mediante elaboración de significados y otras, más complejas, cuando se produce una elaboración basada en la significación de los elementos de la información. Las estrategias de organización consisten en establecer, de un modo explícito, relaciones internas entre los elementos que componen los materiales de aprendizaje y con los conocimientos previos que posea el sujeto. Éstos operan de una doble manera: primero, porque depende de los que el aprendiz posea (cantidad y calidad) el que pueda elaborar de manera más o menos compleja esos materiales y en segundo lugar, porque la estructura cognitiva resultante del nuevo aprendizaje modificará la organización de esos conocimientos previos. Entre las estrategias consideradas de organización se suelen citar las clasificaciones; la construcción de redes de conocimientos (networking); estructuras de nivel superior (covariación, comparación, colección, descripción y respuesta) cada una de las cuales implica una técnica cognitiva específica; los mapas conceptuales; la V de Gowin, etc.

Aproximándose más a los estilos, por cuanto suponen una manera habitual de concebir y tratar la información, que a las estrategias es ya clásica la distinción entre los enfoques sobre los niveles de procesamiento, superficial y profundo. El primero orientado a procurar un incremento cuantitativo de la información y la memorización o repetición literal de ésta mientras que el profundo busca establecer significados $\mathrm{y}$, mediante ellos, 
comprender la información y la realidad. Estos dos enfoques representan una tradición en el pensamiento que se reflejan en la tendencia profundo-significativa frente a la superficial-memorística. Naturalmente en el fondo de estas tendencias, que bien podrían ser estilos de aprendizaje, subyacen orientaciones o disposiciones de los sujetos como las concepciones del aprendizaje que cada uno tenga, su estilo de atribución y de percepción y otros aspectos relacionados con la personalidad.

Hemos descrito someramente las estrategias, sus funciones y clasificación de manera excesivamente analítica. Sin embargo, los comportamientos humanos suceden en una implicación envolvente de los diferentes componentes de la persona de manera que las funciones cognitivas se ven dependientes de otros aspectos de la personalidad como las emociones, el temperamento, la atribución, la autopercepción y otros. Por esta razón, algunos estudiosos de las estrategias incluyen, como una clase más de éstas, las llamadas estrategias de apoyo. Éstas no contribuyen directamente al logro del fin propuesto pero lo posibilitan creando las condiciones que hagan posible la puesta en marcha del plan previsto e incluso la misma posibilidad de establecer el plan. Según Danserau (1985) las estrategias de apoyo son aquéllas que en lugar de dirigirse directamente al aprendizaje de los materiales, tienen como misión incrementar la eficacia de ese aprendizaje mejorando las condiciones en que se produce. Entre ellas se suelen referir estrategias para incrementar la motivación, la atención, la concentración y en general el aprovechamiento de los propios recursos cognitivos. Así pues, vendrían a ser autoinstrucciones para mantener unas condiciones óptimas para la aplicación de las estrategias y, en el caso del aprendizaje escolar pueden ir dirigidas a incrementar la motivación, la autoestima, la atención.

Según ciertas hipótesis y teorías, el uso y la combinación reiterada de ciertas estrategias, la frecuentación de cierto tipo de tareas cognitivas (solución de problemas, razonamiento lógico, análisis, clasificaciones o seriaciones, etc.) así como cierta disposición personal (orientación y estilo personal, estilos cognitivos, estilos perceptivos, etc.) van conformando un perfil de aprendiz que tiene disposición y orientación a usar ciertas estrategias, a percibir y organizar la información de una determinada manera lo que confiere un estilo de aprender. Estas hipótesis sobre estilos de aprendizaje han desencadenado una gran cantidad de investigaciones y desarrollado una estimable cantidad de instrumentos y pruebas que pueden llegar a tener mucha utilidad en la función del autoconocimiento o del conocimiento de las orientaciones de los propios alumnos en la selección y uso de estrategias de aprendizaje y elaboración de planes para aprender.

Finalmente, el espectacular desarrollo de publicaciones tanto sobre aspectos teóricos de las estrategias con elaboraciones y reelaboraciones sucesivas como sobre otros aspectos aplicados tienen en definitiva una finalidad: conocer mejor el proceso de aprender de los individuos y elaborar técnicas de intervención para la mejora del mismo mediante el entrenamiento de los sujetos sobre todo jóvenes. Algunas de las líneas de trabajo actualmente son:

- desarrollo de instrumentos fiables de evaluación y medida de la actividad estratégica de los aprendices. 
- elaboración de técnicas de entrenamiento en el uso de determinadas estrategias para favorecer su uso en los aprendices.

- determinación de la influencia de los entornos de aprendizaje (educación escolar, educación familiar, $\mathrm{EaD}$, educación de adultos, entre otros).

- establecer relaciones entre los contenidos de aprendizaje (matemáticas, ciencias sociales, ciencias naturales y físicas, filosofía, etc.) y la eficacia de estrategias que permitan predecir una mayor o menor facilidad para ciertas tareas y prevenir su entrenamiento específico.

- establecer pautas prescriptivas $u$ orientaciones de aprendizaje conjuntamente con la propuesta de contenidos a aprender una vez establecida la relación positiva entre un determinado entorno de aprendizaje y el estilo o estilos apropiados.

¿Qué aplicación tienen los conceptos sobre estrategias y estilos de aprendizaje al entorno de la Educación a Distancia (EaD)? Reflexionar y sacar algunas conclusiones sobre ello es el verdadero objetivo de este módulo. Recordando y reactualizando a través de la red los conceptos básicos sobre estos aspectos del proceso de aprender, puesto que en definitiva esta información en sustancia es conocida por todos los miembros de este curso, nos servimos de ellos para orientar nuestra reflexión abierta, basada en experiencia propia como aprendiz y educador, original y orientada a promover propuestas de futuro.

Realzar el papel de la metacognición en la EaD resulta algo evidente. El carácter autodidáctico que, en gran medida, conserva este entorno parece requerir, más que ningún otro, de un buen conocimiento de los propios recursos. Pero, ¿es posible o fácil inducir elementos que incrementen esta ineludible e intransferible función del aprendiz de EaD? ¿Cómo o con qué recursos? ¿Existen experiencias en este sentido? ¿Bajo que criterios o condiciones se podría intentar incorporar en las actividades de EaD de algunos de nosotros elementos de control de la actividad cognitiva de los sujetos? ¿Una buena guía didáctica puede cumplir esas funciones? ¿Bajo qué criterios o condiciones?

En cuanto a los estilos, ¿seríamos capaces -no sabemos si también osados- de definir los rasgos de un estilo de aprendizaje ideal para la $\mathrm{EaD}$ ? ¿O bien una combinación de otros siguiendo la clasificación del documento sobre estilos (características de los cuatro estilos según nuestra perspectiva)? ¿Es necesario ajustar los estilos de aprendizaje y los estilos de enseñanza? ¿Es al menos necesario tenerlos en cuenta? ¿Existe suficiente cultura en la dirección de tratar de observar y conocer el estilo de aprender de los alumnos para orientar la enseñanza en la dirección de mejorar las condiciones de aprendizaje? ¿Cuáles pueden ser ingredientes esenciales del entorno de aprendizaje EaD para llevar a cabo esa función? ¿Habría que destinar algún material y sesión a orientar a los futuros aprendices de un entorno EaD sobre su propia observación y conocimiento de su estilo de aprender y/o ofrecerles instrumentos psicológicos que le ayuden a observarse (test, cuestionarios, protocolos de observación? ¿Conocemos experiencias sobre esto? 
De esta forma se nos suscitan varias interrogantes en relación con el eLearning y los EVAs: Al igual que nos planteábamos en su momento si el concepto de objeto de aprendizaje reutilizable es compatible con los requisitos de interdependencia de contenidos de aprendizaje, nos podemos plantear ahora si el concepto de objeto de aprendizaje y el subsiguiente de la reusabilidad es compatible con cualquier estilo de aprendizaje: ¿hay estilos más privilegiados que otros y cuales son?; ¿Qué requisitos se tendrían que asegurar para la mayoría de estilos fuesen compatibles y cómo se tendría que repercutir en especificaciones de learning design para que la exclusión fuese la mínima? ¿No tendríamos pues que plantearnos de forma necesaria cómo se pueden o se deben trasladar criterios de compatibilidad de los distintos etilos y estrategias con los objetos de aprendizaje y cómo se deben trasladar en la fase de diseño y de elaboración, cómo deben operar en la fase de ejecución? ¿Qué datos, informaciones y criterios se añadan a las informaciones de otro tipo que se adjuntan a los objetos de aprendizaje en los metadata? ¿Se hace como apoyo a la intervención educativa o de forma automatizada (si es posible)?

Y además tendremos que plantear estas cuestiones como requisitos de calidad derivados de los principios y teorías sobre estilos estrategias y metacognición en general pero aplicados a learning objects, en relación con la existencia en el diseño instruccional de pautas prescriptivas u orientaciones de aprendizaje conjuntamente con la propuesta de contenidos a aprender una vez establecida la relación positiva entre un determinado entorno de aprendizaje y el estilo o estilos apropiados. Estos criterios nos ofrecen pues unas pautas operativas para definir la singularidad cognitiva de los objetos de aprendizaje reutilizables. El proceso es complejo puesto que la secuencia o la presentación de los contenidos no siempre se corresponderá necesariamente con la "secuencia o con la organización que nos parezca lógica y natural de los contenidos" y la secuenciabilidad o la inclusión en estrategias podría tener como origen criterios distintos de análisis sobre cómo se forman los conceptos y las ideas o cómo se pueden organizar. Incluso podría concluirse que HAY objetos de aprendizaje no reutilizables para según qué estilos o de unas estrategias a otras. Esta sería pues la última ratio de la cuestión de la calidad en EVA. Y el diseño educativo sería en cualquier caso la respuesta.

Sirvan pues estas preguntas para favorecer una reflexión orientada al diseño educativo y a la calidad los entornos de aprendizaje, que es el principal propósito de este documento.

Conclusión del artículo en la versión original: 1 de octubre de 2007 Aprobación del artículo: 15 de octubre de 2007 Publicación de la versión original: 31 de enero de 2008

Esteban, M., y Zapata, M. (2016). Estrategias de aprendizaje y eLearning. Un apunte para la fundamentación del diseño educativo en los entornos virtuales de aprendizaje. Consideraciones para la reflexión y el debate. Introducción al estudio de las estrategias y estilos de aprendizaje. RED. Revista de Educación a Distancia, 50. Consultado (día/mes/año) en http://www.um.es/ead/red/50 


\section{Referencias}

Danserau, D. (1985). Learning strategy research, en Segal, J., Chipman, S. Y Glaser, R (eds), Thinking and learning skills, vol. 1. Hilldale, New Yersey: L. Erlbaum.

Danserau, D. (1985). Learning Strategies Research. En J. W. Segal, S. F. Chipman y R. Glaser (eds.). Thinking and Learning Skills. Vol. 1, pp. 209-240. Hillsdale, NJ: Erlbaum.

Danserau, D. (1988). Cooperative learning strategies. En C.M. Weinstein, E.T.Goetz y P.A. Alexander (Eds.). Learning and study strategies: Issues in assessment, instruction and evaluation. San Diego: Academic Press.

Esteban, M. (2003, Febrero). Las estrategias de aprendizaje en el entorno de la Educación a Distancia. Consideraciones para la reflexión y el debate. Introducción al estudio de las estrategias y estilos de aprendizaje. RED. Revista de Educación a Distancia. $N^{o}$ 7. http://www.um.es/ead/red/7/estrategias.pdf. Revisado el 12/01/2008 9:42

Zapata, M. (2005, Febrero). Secuenciación de contenidos y objetos de aprendizaje. RED. Revista de Educación a Distancia, número monográfico II. http://www.um.es/ead/red/M2/zapata47.pdf . Revisado el 12/01/2008.

Zapata, M. y Lizenberg, N. (2006, Junio). SEQUENCING OF CONTENTS AND LEARNING OBJECTS - part III. SECUENCIACION DE CONTENIDOS Y OBJETOS DE APRENDIZAJE (III)). RED. Revista de Educación a Distancia, número monográfico II. http://www.um.es/ead/red/M2/ . Revisado el 12/01/2008.

Zapata, M. y Cerezo, F. (2007, Mayo).La formación continua en Cataluña: Formación de formadores. Conclusiones y propuestas a partir de los resultados del estudio Formación de Formadores y competencias transversales. RED. Revista de Educación a Distancia. $N^{o} 17$. http://www.um.es/ead/red/17/ Revisado el $12 / 01 / 2008$.

WILEY, D. A. (2002). "Connecting Learning Objects to Instructional Design Theory: A Definition, a Metaphor, and a Taxonomy.” The Instructional Use of Learning Objects. Bloomington, IN: Agency for Instructional Technology.

\footnotetext{
${ }^{\text {i }}$ Griffiths, D. Blat, J. Garcia, R. Sayago, S. (2005, Junio). La aportación de IMS Learning Design a la creación de recursos pedagógicos reutilizables. RED. Revista de Educación a Distancia, número monográfico II. Consultado 02/07/2006, en http://www.um.es/ead/red/M5/.

ii IMS Global Learning Consortium (2001). IMS Guidelines for Developing Accessible Learning. Applications. Version 0.6 White Paper 3. Principles for Accessibility in Online Distributed Learning. Consultado el 02/07/2006, en http://www.imsglobal.org/accessibility/accwpv0p6/ imsacc_wpv0p6.html\#1272423
} 


\section{Principles for Accessibility in Online Distributed Learning}

The following principles represent best practices for producing accessible software applications and content for online distributed learning. These principles primarily address accessibility for people who have sensory or mobility disabilities, and to a lesser extent on the wide array of accessibility issues faced by people with cognitive disabilities. Many of these principles will also be beneficial to users with cognitive disabilities, such as a learning disability, however comprehensive solutions for these users goes beyond the scope of this document. Attention should be given to implementing these practices from the beginning of the design/development process, since retrofitting a product or content for accessibility is almost always significantly more labor-intensive and more costly than incorporating it from the start.

The Six Principles:

1. Allow for customization based on user preference.

2. Provide equivalent access to auditory and visual content based on user preference.

3. Provide compatibility with assistive technologies and complete keyboard access.

4. Provide context and orientation information.

5. Follow IMS specifications and other relevant specifications, standards, and/or guidelines.

6. Consider the Use of XML (Extensible Mark-up Language). 